\title{
GADD45B Facilitates Metastasis of Ovarian Cancer Through Epithelial-Mesenchymal Transition
}

This article was published in the following Dove Press journal: OncoTargets and Therapy

\author{
Lanqing Gong $\mathbb{D}^{1, *}$ \\ Liqiong Cai (D) ${ }^{1} *$ \\ Guodong $\mathrm{Li}^{2}$ \\ Jing Cai ${ }^{\prime}$ \\ Xiaoqing $\mathrm{Yi}^{1}$
}

'Department of Obstetrics and Gynecology, Union Hospital, Tongji Medical College, Huazhong University of Science and Technology, Wuhan 430022, People's Republic of China; ${ }^{2}$ Cancer Research Institute, Tongji Hospital, Huazhong University of Science and Technology, Wuhan 430022, People's Republic of China

*These authors contributed equally to this work
Correspondence: Xiaoqing Yi Department of Obstetrics and Gynecology, Union Hospital, Tongji Medical College, Huazhong University of Science and Technology, 1277 Jiefang Avenue, Wuhan, Hubei Province 430022, People's Republic of China

Tel/Fax: +862785351649

Email yxq1987724@I26.com
Background: Growth arrest and DNA-damage-inducible 45 beta $(G A D D 45 B)$ is overexpressed and is associated with poor clinical outcomes in many human cancers, but the clinical implication of $G A D D 45 B$ in epithelial ovarian cancer (EOC) remains unclear.

Methods: Bioinformatics analysis of The Cancer Genome Atlas (TCGA) and gene expression omnibus (GEO) cohorts was used to illustrate the relationship between $G A D D 45 B$ expression and metastasis, as well as the survival time of EOC. GADD45B was downregulated by siRNAs in EOC cells, and migration ability was determined by a transwell assay and wound-healing assay. Kyoto Encyclopedia of Genes and Genomes (KEGG) pathway enrichment analysis and gene set enrichment analysis (GSEA) were conducted to discover the downstream pathway of $G A D D 45 B$. The regulation of epithelial-mesenchymal transition (EMT) by GADD45B was verified by Western blotting and qRT-PCR. Finally, the correlation of $G A D D 45 B$ expression with EOC metastasis was investigated in EOC tissues by immunohistochemistry.

Results: Overexpression of $G A D D 45 B$ indicates shorter overall survival time and progression-free survival time, and it is an independent risk factor for poor survival in EOC patients. Elevated $G A D D 45 B$ is related to venous invasion, lymphatic invasion and peritoneal carcinomatosis. Downregulation of GADD45B decreases the migration of ES2 and SKOV3 cells. Further KEGG enrichment analysis and GSEA revealed that EMT may be the downstream pathway of GADD45B. In addition, reduced GADD45B increases the expression of E-cadherin and decreases that of $\mathrm{N}$-cadherin and vimentin. Finally, immunohistochemical analysis of GADD45B expression revealed that the expression of GADD45B in omental metastatic tissues was higher than that in matched primary ovarian cancer tissues. These results suggest that elevated GADD45B promotes the motility of ovarian cancer cells through EMT and is associated with EOC metastasis.

Conclusion: GADD45B can promote the motility of ovarian cancer cells through EMT, is associated with EOC metastasis, and may be a new biomarker of metastasis and prognosis.

Keywords: epithelial ovarian cancer, GADD45B, metastasis, epithelial-mesenchymal transition

\section{Introduction}

Ovarian cancer has the highest mortality of gynaecological cancers. ${ }^{1}$ Despite improved treatment in recent years, as many as 185,000 patients die worldwide every year because of the late tumour stage and tumour metastasis. ${ }^{2,3}$ Epithelial ovarian cancer (EOC) is the most common subtype among ovarian cancers, 
accounting for $90 \%$ of all cases. EOCs are classified as serous $(52 \%)$, endometrioid $(10 \%)$, mucinous $(6 \%)$, or clear cell $(6 \%)$ by tumour cell histology, with one-quarter of EOCs classified as rare or unspecified subtypes. ${ }^{4}$ Metastasis is one of the main reasons for the poor prognosis of EOC. ${ }^{5,6}$ Therefore, it is necessary to find new biomarkers to predict tumour metastasis and poor disease prognosis, which deepens our understanding of EOC tumorigenesis, progression, invasion and metastasis and contributes to oncotherapy.

The growth arrest and DNA damage-inducible 45 beta $(G A D D 45 B)$ gene is a member of the growth arrest and DNA damage-inducible 45 (GADD45) gene family, including $G A D D 45 A, G A D D 45 B$ and $G A D D 45 G$. It has been implicated in stress signalling in response to physiological and environmental stress, including oncogenic stress, which can result in cell cycle arrest, DNA repair, cell survival, senescence, and apoptosis. ${ }^{7}$ GADD $45 B$ responds to environmental stresses by activating the p38/ JNK pathway. This activation is mediated via its protein binding and activation of MTK1/MEKK4 kinase. ${ }^{8}$ Moreover, $G A D D 45 B$ is an epigenetic regulator of juvenile social behaviour by altered epigenetic programming of $\alpha_{2}$-adrenoceptor and local proinflammatory cytokine production in the rodent amygdala. ${ }^{9}$ In addition, $G A D D 45 B$ correlates closely with tumour prognosis and progression; for example, high expression levels of $G A D D 45 B$ are an independent prognostic factor of decreased OS and PFS in stage II colorectal cancer (CRC) patients, ${ }^{10}$ while increased expression levels of $G A D D 45 B$ are an independent prognostic marker in papillary thyroid carcinoma patients treated with total thyroidectomy and radioiodine therapy. ${ }^{11} G A D D 45 B$ determines chemoresistance and invasive growth of side population cells of human embryonic carcinoma, ${ }^{12}$ and silencing of $G A D D 45 B$ reduces the invasion and migration of cholangiocarcinoma cells. ${ }^{13}$ These results suggest that GADD45B may contribute to the malignant phenotypes of cancer. However, the underlying mechanism is not yet fully characterized.

Despite the important role of $G A D D 45 B$ in tumours, its function in EOC development has not been elucidated clearly. Bioinformatics analysis of gene expression data from The Cancer Genome Atlas database (TCGA) indicated a prognostic gene set of 25 candidate genes, including $G A D D 45 B$, which was significantly associated with EOC prognosis. ${ }^{14}$ Xinguo Lu integrated different levels of genomics datasets from TCGA, including gene expression, copy number and somatic mutation, to identify drivers of resistance and sensitivity to anticancer drugs and reported that $G A D D 45 B$ is a candidate modulator of the drug response to cis-platinum in EOC. ${ }^{15}$ Some researchers found that high $G A D D 45 B$ levels resulted in shorter recurrence-free survival in the GSE9891 EOC dataset, and TME-specific $G A D D 45 B$ loss in ID8-Luc ovarian adenocarcinoma allografts augmented proinflammatory tumour-associated macrophage (TAM) activation and intratumoural T-cell infiltration while reducing ovarian oncogenesis. ${ }^{16}$ Extending these studies, in the present study, bioinformatics analysis of TCGA and gene expression omnibus (GEO) datasets confirmed that high expression of GADD45B in EOC correlated with metastasis and poor patient survival. Moreover, the function and mechanism of $G A D D 45 B$ in EOC cell metastasis were revealed by bioinformatics analysis and in vitro analysis.

\section{Materials and Methods Bioinformatics Analysis}

Level 3 HiSeq RNA-seq data were downloaded from The Cancer Genome Atlas (TCGA, https://tcga-data.nci.nih. gov/tcga/) website for 412 EOC samples. In addition, GSE49997, ${ }^{17}$ another human expression array and clinical information, was downloaded from the Gene Expression Omnibus (GEO, http://www.ncbi.nlm.nih.gov/geo/) website because of the relatively large number of samples and complete information about survival and tumour metastasis. The relationship between $G A D D 45 B$ and the clinicopathologic features of EOC in these datasets was studied by analysis of variance (ANOVA). To determine the prognostic significance of $G A D D 45 B$ in EOC patients, we used the online microarray data analysis website KMPlotter $^{18}$ to investigate the prognostic significance of $G A D D 45 B$ in EOC datasets (TCGA, GSE9891, GSE26712, GSE26193). "GADD45B" was entered as a gene symbol, and the probe "207574_s_at" and the "Auto select best cut off" button were selected. KaplanMeier and Log rank tests were used to evaluate the difference in overall survival time (OS) and progression-free survival time (PFS). Kaplan-Meier and Log rank tests were also performed on the downloaded TCGA RNASeq data. The mRNA expression of GADD45B between EOC with/without metastasis and different FIGO stages or grades was compared with Student's $t$-test or one-way analysis of variance in TCGA RNASeq data and microarray data GSE49997. Furthermore, univariate and 
multivariate Cox regression analyses of overall survival in the TCGA EOC cohort were performed to illustrate the correlation between $G A D D 45 B$ upregulation and poor survival. To further reveal the molecular mechanisms of $G A D D 45 B$ in EOC metastasis, Kyoto Encyclopedia of Genes and Genomes (KEGG) pathway enrichment analysis and gene set enrichment analysis (GSEA) were carried out. First, we used Pearson correlation to calculate the coexpression of $G A D D 45 B$ with other genes in the TCGA cohort. Circos plots were then draw by $\mathrm{R}$ language. GSEA was carried out by the Database for Annotation, Visualization and Integrated Discovery (DAVID) online website, and the "hallmark gene sets" were used for running GSEA. P $<0.05$ and FDR $\mathrm{Q}<$ 0.25 were set as the default parameters to generate enrichment results.

\section{Cell Culture}

A2780, CAOV3, C13*, ES2, HO8910, OV2008 and SKOV3 OC cell lines were purchased from the China Centre for Type Culture Collection (CCTCC, Wuhan, China). Among them, A2780 is a human ovarian cancer cell line, CAOV3 is a human papillary ovarian adenocarcinoma cell line, $\mathrm{C} 13^{*}$ is a human ovarian cancer cell line, ES2 is a human clear ovarian cell line, HO8910 is a human ovarian cancer cell line, OV2008 is a human ovarian cancer cell line, and SKOV3 is a human ovarian adenocarcinoma cell line. All cells were cultured at $37^{\circ} \mathrm{C}$ with DMEM/F12 medium (Gibco, Grand Island, NY, USA) containing $10 \%$ foetal calf serum (Gibco, USA) under a $5 \% \mathrm{CO}_{2}$ atmosphere. Cell lines were authenticated by short tandem repeat (STR) profiling and confirmed to be mycoplasma negative.

\section{Transfection in vitro}

$G A D D 45 B$ siRNA and a scramble nontargeting siRNA (siNC) were purchased from Gene Pharma (Suzhou, China). The $G A D D 45 B$ siRNA sequence was as follows: si-GADD45B-1, sense 5'-CGGCCAAGUUGAUGAAU GUTT-3' and antisense 5'-ACAUUCAUCAACUUGGCC GTT-3'; si-GADD45B-2, sense 5'-GCUGUGACAACG ACAUCAATT-3' and antisense 5'-UUGAUGUCGUUG UCACAGCTT-3'; si-GADD45B-3, sense 5'-CCAGCU ACUGCGAAGAAAGTT-3' and antisense 5'-CUUUCUU CGCAGUAGCUGGTT-3'. Transient transfection of siRNA was conducted using Lipofectamine 2000 transfection reagent (Invitrogen, Carlsbad, CA, USA) according to the manufacturer's instructions.

\section{Quantitative Real-Time PCR (qRT-PCR)}

Total RNA was extracted from cells using TRIzol reagent (TaKaRa) according to the instructions. After measuring the RNA concentration with a Nanodrop 2000, reverse transcription was performed using Superscript III RT (TaKaRa). Quantitative real-time PCR was performed on an Applied Biosystem StepOne Plus PCR system (ABI) using SYBR Green PCR Master Mix (TaKaRa). All primers were designed based on NCBI reference sequences and synthesized by Invitrogen (Suzhou, China). Primer sequences were listed in Supplemental Table S1. Target gene expression was calculated using the $2^{-\Delta \Delta \mathrm{Ct}}$ method. Each experiment was performed in triplicate at least three times.

\section{Western Blotting Analysis}

Total cell protein was extracted with radioimmunoprecipitation assay (RIPA) buffer (Beyotime Biotechnology, Shanghai, China) including a protease inhibitor cocktail. The protein concentration was measured with a bicinchoninic acid (BCA) assay, and then samples were separated by $10 \%$ sodium dodecyl sulfate-PAGE (SDSPAGE) and transferred onto a PVDF membrane. The membranes were blocked with $5 \%$ nonfat milk in Tris-buffered saline with Tween-20 (TBST) for 1 hour at room temperature and incubated with primary antibodies against $\beta$-actin (1:5000 dilution, Proteintech, USA), GADD45B (1:200 dilution, Affinity Biosciences, China), E-cadherin (1:1000 dilution, CST, USA), N-cadherin (1:1000 dilution, CST, USA) and vimentin (1:2000 dilution, CST, USA) at $4^{\circ} \mathrm{C}$ overnight. Horseradish peroxidase-conjugated anti-rabbit or anti-mouse secondary antibodies (1:5000 dilution, CST, USA) were subsequently used. Protein bands were detected with an enhanced chemiluminescence kit (Pierce, Thermo Scientific, Waltham, MA, USA) in a Molecular Imager ${ }^{\circledR}$ ChemiDocTM XRS+ with Image $\mathrm{Lab}^{\mathrm{TM}}$ Software (BioRad Laboratories, Hercules, CA, USA).

\section{Transwell Migration Assay}

Forty-eight hours after siRNA transfection, $8 \times 10^{4}$ cancer cells were plated in 24-well Transwell plates with inserts $(8 \mu \mathrm{m}$ in diameter, Corning). The medium in the inserts was free of FBS, whereas the medium outside the inserts was supplemented with $10 \%$ FBS. After 24 hours of incubation, the cell inserts were fixed with formaldehyde, stained with $0.1 \%$ crystal violet, observed and photographed under a microscope. The experiment was repeated at least three times. 


\section{Wound-Healing Assay}

Forty-eight hours after siRNA transfection, cells were seeded in 6-well plates to approximately $100 \%$ confluence. A linear scratch wound was created in the confluent monolayer using a $200-\mu \mathrm{L}$ pipette tip. After washing the cells gently with phosphate buffer solution (PBS) to remove suspended cells, the culture medium was replaced with serum-free medium to block cell proliferation, and cancer cells were allowed to close the wound for 24 hours. The closure area was determined by comparing the images from the start time point $(0 \mathrm{~h})$ to the last time point $(24$ h) under microscopy using Image-Pro Plus 6 software. The experiment was repeated three times.

\section{Cell Counting Kit-8 (CCK8) Cell Viability Assay}

The viability abilities of cells were analysed according to the manufacturer's instructions for CCK8 (DOJINDO, Japan). First, 48 hours after siRNA transfection, the transfected SKOV3/ES2 cells were resuspended and inoculated into a $96-$ well plate at 5000 cells $/ 100 \mu \mathrm{L}$ per well, with 5 wells per set. After 24 hours cultured, ten microlitres of CCK8 solution was added to incubation for 4 hours, the absorbance at $450 \mathrm{~nm}$ was measured with a microplate reader. The experiment was repeated three times.

\section{EdU (5-Ethynyl-2'-Deoxyuridine) Proliferation Assay}

An EdU proliferation assay was also used to investigate the proliferation of cells according to the manufacturer's instructions for the EdU proliferation kit (RIBOBIO, China). Forty-eight hours after siRNA transfection, the transfected SKOV3/ES2 cells were inoculated into 96well plates at 10,000 cells per well. After the cells were attached to the wall, EdU solution was incubated for 2 hours for EdU labelling, and then the cells were fixed with paraformaldehyde for 30 minutes. After Apollo staining, $1 \times$ Hoechst 33342 reaction solution was used for DNA staining. Images were taken under a fluorescence inverted microscope within 48 hours after staining and counted with ImageJ software. The experiment was repeated three times.

\section{Immunohistochemistry (IHC)}

For IHC analysis, $4 \mu \mathrm{m}$ sections of paraffin-embedded human tissue specimens were performed by a PV-9003 Kit (ZSGBBIO, China) according to the manufacturer's instructions. In brief, after three rounds of TO-type biotablet transparent agent dewaxing, dehydration by gradient ethanol, and antigen retrieval in $0.01 \mathrm{M}$ citrate solution at $95^{\circ} \mathrm{C}$ for 20 minutes, the samples were treated with $3 \%$ $\mathrm{H}_{2} \mathrm{O}_{2}$ at room temperature for 20 minutes to remove the effect of endogenous peroxidase, then blocked with normal goat serum for 20 minutes at room temperature. Tissue sections were incubated with the primary antibody against GADD45B (1:250 dilution, Abcam, ab105060, UK) overnight at $4^{\circ} \mathrm{C}$. Incubation with secondary antibody labelled with horseradish peroxidase polymer was carried out for 30 minutes at room temperature. Finally, the staining processes were performed with DAB chromogenic solution (AR1022, BOSTER, China), and haematoxylin (AR0005, BOSTER, China) was used to counterstain the nuclei. Rabbit IgG was used as an isotype control. Images were captured with a microscope and assessed with ImagePro Plus as mean density = integrated optical density/area of DAB staining. ${ }^{19}$

\section{Statistical Analysis}

Statistical analysis was performed using SPSS 17.0 statistical software and GraphPad Prism 5.0 software. Numerical data are expressed as the mean \pm standard deviation. Student's $t$-test was used to compare the differences between the two groups. $\mathrm{P}<0.05$ was considered significant.

\section{Results}

\section{Overexpression of GADD45B Indicates Poor EOC Survival}

To investigate the prognostic significance of $G A D D 45 B$ in EOC patients, we used the online analysis website KMPlotter. In summation of different microarray datasets, overexpression of $G A D D 45 B$ indicated shorter overall survival (OS) time and progression-free survival (PFS) time in EOC patients (hazard ratio 1.19, 95\% confidence interval 1.05-1.35, $\mathrm{P}$ value 0.0082 and hazard ratio 1.18 , $95 \%$ confidence interval 1.04-1.35, $\mathrm{P}$ value 0.01 ) (Figure 1A). For individual datasets, high expression of $G A D D 45 B$ portended poor OS and PFS in microarray datasets TCGA (Figure 1B), GSE9891 (Figure 1C), GSE26712 (Figure 1D) and GSE26193 (Figure 1E). Similar results were found in the downloaded RNAsequencing data TCGA RNASeqV1 (Figure 1F). The percentage of EOC samples with high expression of GADD45B in TCGA cohort is showed in Table 1. 

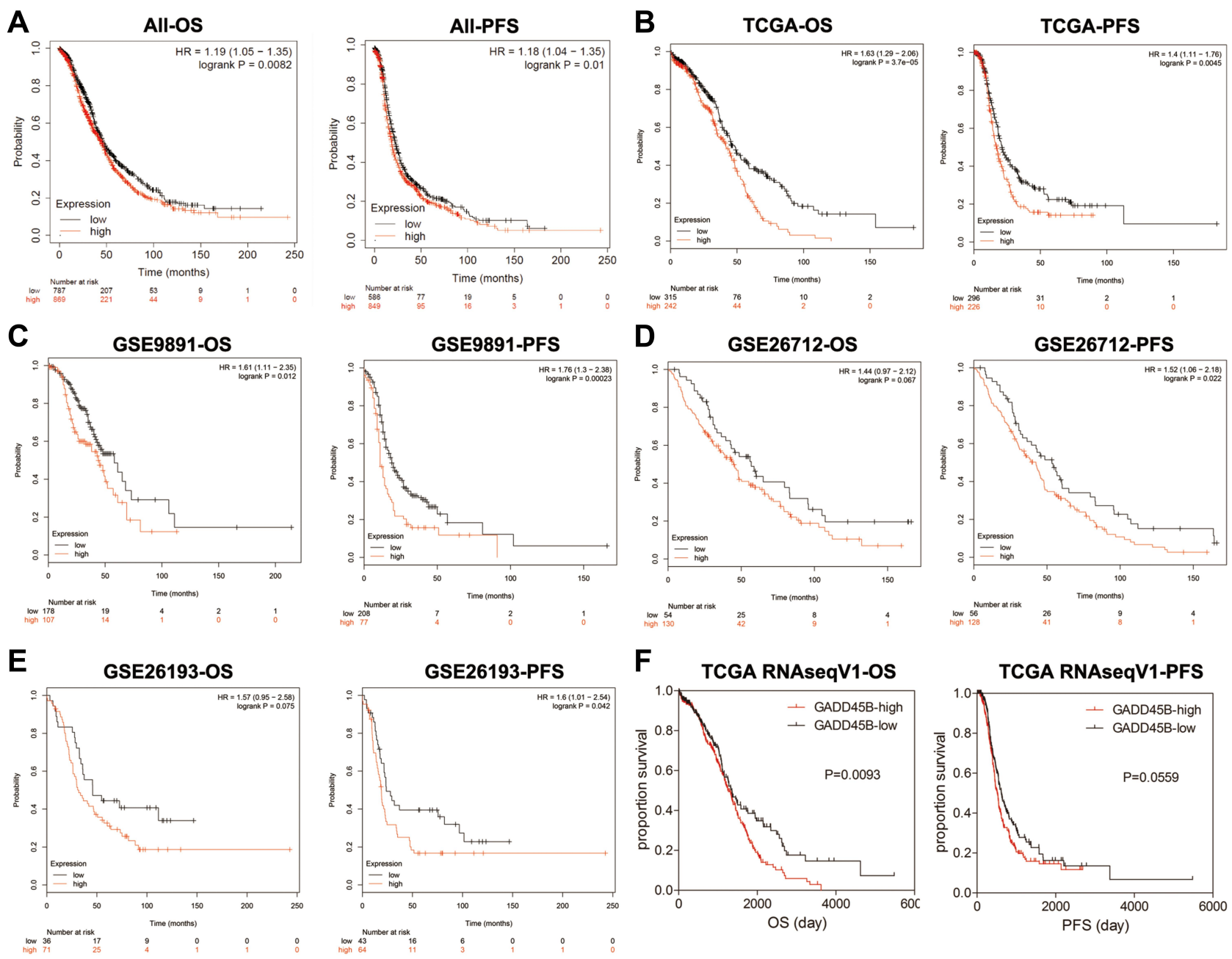

TCGA RNAseqV1-PFS

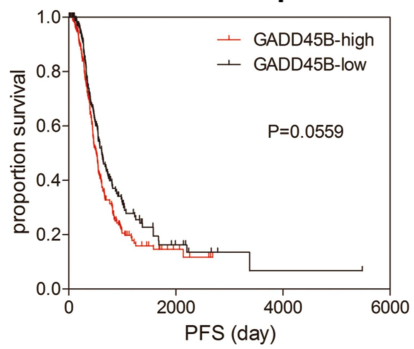

Figure I Overexpression of GADD45B indicates poor EOC survival. Survival analysis in different microarray datasets was conducted based on the expression of GADD45B. (A) Kaplan-Meier survival curves for OS and PFS in summation of different microarray datasets based on the expression of GADD45B. (B) Kaplan-Meier survival curves for OS and PFS in the TCGA cohort. (C) Kaplan-Meier survival curves for OS and PFS in dataset GSE989I. (D) Kaplan-Meier survival curves for OS and PFS in dataset GSE267I2. (E) Kaplan-Meier survival curves for OS and PFS in dataset GSE26193. (F) Kaplan-Meier survival curves for OS and PFS in the downloaded TCGA RNA-seq data.

Further univariate and multivariate Cox regression analyses of OS time in the TCGA ovarian cancer cohort illustrated that $G A D D 45 B$ was an independent risk factor for poor survival in EOC (hazard ratio 1.398, 95\% confidence interval 1.068-1.829, $\mathrm{P}$ value 0.015) (Table 2).

\section{High GADD45B mRNA Levels are Related to Metastasis of EOC}

The relationships between $G A D D 45 B$ mRNA levels and clinicopathological features were further analysed in the TCGA cohort. Elevated expression of GADD $45 B$ was significantly associated with high FIGO stage, venous invasion and lymphatic invasion, but not histologic grade, regardless of RNA sequence data (Figure 2A-D) or microarray data (Figure 2E-H). Compared with EOC tissues without peritoneal carcinomatosis, $G A D D 45 B$ seemed to be overexpressed in those with peritoneal carcinomatosis in the GSE49997 cohort (Figure 2I). The correlation between the mRNA expression of GADD45B and the clinicopathological features is provided in Table 1 . These results were in accordance with the results of the survival analysis.

\section{GADD45B Knockdown Inhibits the Migration of EOC Cells}

To further study the biological function of $G A D D 45 B$ in EOC cells, siRNAs were used to knock down GADD45B expression levels. First, the level of GADD45B in seven ovarian cancer cell lines was tested by Western blotting and qRT-PCR, and ES2 and SKOV3 cells were chosen to conduct the following experiments because of their relatively high expression (Figure 3A and B). The silencing efficiency was validated by qRT-PCR (Figure 3C) and Western blotting (Figure 3D). 
Table I Correlation of GADD45B with Clinicopathological Features in EOC Patients of TCGA Cohort

\begin{tabular}{|c|c|c|c|c|}
\hline \multirow{2}{*}{$\begin{array}{l}\text { Clinicopathological } \\
\text { Features }\end{array}$} & \multirow[t]{2}{*}{$\mathbf{n}$} & \multicolumn{2}{|c|}{ GADD45B } & \multirow[t]{2}{*}{ P value } \\
\hline & & High (\%) & Low (\%) & \\
\hline Age (year) & & & & 0.144 \\
\hline$>65$ & 128 & $64(50.0)$ & $64(50.0)$ & \\
\hline$\leq 65$ & 284 & $164(57.7)$ & $120(42.3)$ & \\
\hline FIGO stage & & & & $0.011 *$ \\
\hline II & 21 & $7(33.3)$ & $14(66.7)$ & \\
\hline III & 326 & $179(54.9)$ & I $47(45.1)$ & \\
\hline IV & 62 & $41(66.1)$ & $21(33.9)$ & \\
\hline Unknown ${ }^{b}$ & 3 & I & 2 & \\
\hline Histologic grade & & & & 0.685 \\
\hline GI-2 & 50 & $26(52.0)$ & $24(48.0)$ & \\
\hline G3 & 356 & $196(55.1)$ & $160(44.9)$ & \\
\hline Unknown ${ }^{\mathrm{b}}$ & 6 & 4 & 2 & \\
\hline \multicolumn{5}{|l|}{ Tumor size } \\
\hline$>1 \mathrm{~cm}$ & 283 & $163(57.6)$ & $120(42.4)$ & 0.189 \\
\hline$\leq \mathrm{Icm}$ & 100 & $50(50.0)$ & $50(50.0)$ & \\
\hline Unknown & 29 & 15 & 14 & \\
\hline Venous invasion & & & & $0.002 *$ \\
\hline Yes & 64 & $35(54.7)$ & $29(45.3)$ & \\
\hline No & 47 & $12(25.5)$ & $35(74.5)$ & \\
\hline Unknown ${ }^{\mathrm{b}}$ & 301 & 181 & 120 & \\
\hline Lymphatic invasion & & & & 0.083 \\
\hline Yes & 105 & $58(55.2)$ & $47(44.8)$ & \\
\hline No & 54 & $22(40.7)$ & $32(59.3)$ & \\
\hline Unknown ${ }^{\mathrm{b}}$ & 253 & 148 & 105 & \\
\hline
\end{tabular}

Notes: ${ }^{a}$ Fisher's exact tests. 'Statistics did not include Unknown group. *P values less than 0.05 were considered statistically significant. Boldface type indicates significant values. Split point for GADD45B mRNA was determined by ROC curve. Abbreviations: FIGO, International Federation of Gynecology and Obstetrics; TCGA, The Cancer Genome Atlas.

Wound-healing assays revealed that knockdown of GADD45B decreased the migration abilities of ES2 and SKOV3 cells (Figure 3E-H). Moreover, the number of migrated ES2 and SKOV3 cells significantly decreased after GADD45B siRNA transfection (Figure 3I-K). Thus, GADD45B knockdown inhibited the migration capacity of EOC cells. To confirm whether the changes in motility when siRNA was knocked down GADD45B expression were due to decreased cell proliferation and viability, we verified cell proliferation and viability through EdU proliferation experiments and CCK-8 experiments, respectively. The results showed that compared with the NC group, there was no difference in the cell proliferation ability of the siRNA-downregulated groups (Figure 4A and B). The cell viability of the siRNAdownregulated groups was higher than that of the NC group
Table 2 Multivariate Cox Regression Analyses in TCGA Cohort

\begin{tabular}{|c|c|c|c|c|}
\hline \multirow[t]{3}{*}{ Variables } & \multicolumn{4}{|l|}{ os } \\
\hline & \multicolumn{2}{|l|}{ Univariate } & \multicolumn{2}{|l|}{ Multivariate } \\
\hline & $\begin{array}{l}\text { HR }(95 \% \\
\text { CI) }\end{array}$ & p value & $\begin{array}{l}\text { HR }(95 \% \\
\text { CI) }\end{array}$ & p value \\
\hline $\begin{array}{l}\text { Age }(>65 y / \\
\leq 65 y)\end{array}$ & $\begin{array}{l}\text { I.345 } \\
(1.032-1.752\end{array}$ & 0.028 & $\begin{array}{l}1.358 \\
(1.041-\mid .772)\end{array}$ & 0.024 \\
\hline $\begin{array}{l}\text { Stage (III- } \\
\text { IV/I-II) }\end{array}$ & $\begin{array}{l}2.101 \\
(0.989-4.464)\end{array}$ & 0.053 & $\begin{array}{l}\text { I.875 } \\
(0.880-3.996)\end{array}$ & 0.104 \\
\hline $\begin{array}{l}\text { Grade (3/ } \\
\text { I-2) }\end{array}$ & $\begin{array}{l}1.343 \\
(0.912-1.978)\end{array}$ & 0.135 & - & - \\
\hline $\begin{array}{l}\text { Tumor size } \\
(>\mathrm{Icm} / \\
\leq \mathrm{Icm})\end{array}$ & $\begin{array}{l}0.985 \\
(0.729-1.332)\end{array}$ & 0.921 & - & - \\
\hline $\begin{array}{l}\text { Residual } \\
\text { tumor size } \\
(>1 \mathrm{~cm} / \\
\leq \mathrm{lcm})\end{array}$ & $\begin{array}{l}\text { I.234 } \\
(0.924-1.649)\end{array}$ & 0.154 & - & - \\
\hline $\begin{array}{l}\text { GADD45B } \\
\text { (High/Low) }\end{array}$ & $\begin{array}{l}1.423 \\
(1.089-1.859)\end{array}$ & 0.010 & $\begin{array}{l}1.398 \\
(1.068-1.829)\end{array}$ & 0.015 \\
\hline
\end{tabular}

Notes: $\mathrm{P}$ values less than 0.05 were considered statistically significant. Boldface type indicates significant values. Split point for GADD45B mRNA from database was determined by ROC curve.

Abbreviations: $\mathrm{Cl}$, confidence interval; $\mathrm{HR}$, hazard ratio; OS, overall survival; TCGA, The Cancer Genome Atlas.

(Figure 4C). These results indicate that the motility changes caused by GADD45B silencing may not be due to decreases in cell proliferation and viability.

\section{GADD45B Correlates with the Epithelial- Mesenchymal Transition of EOC}

To further understand the molecular mechanisms underlying how $G A D D 45 B$ affects the migration of EOC cells, KEGG enrichment analysis was carried out in RNA-sequencing data from the TCGA EOC cohort (412 patients with primary ovarian cancer). A total of 1995 genes correlated with $G A D D 45 B$ (Pearson $r>0.3$ or $<-0.3$ ) (Figure 5A). Next, KEGG enrichment analysis was implemented by the DAVID bioinformatics tools. The KEGG results provided significant pathway information for GADD45B coexpressed genes, including Staphylococcus aureus infection, malaria, cell cycle, ECM-receptor interaction, focal adhesions, and the TNF signalling pathway (Figure 5B). GSEA of the TCGA cohort showed that $G A D D 45 B$ expression was strongly related to the EMT 
A

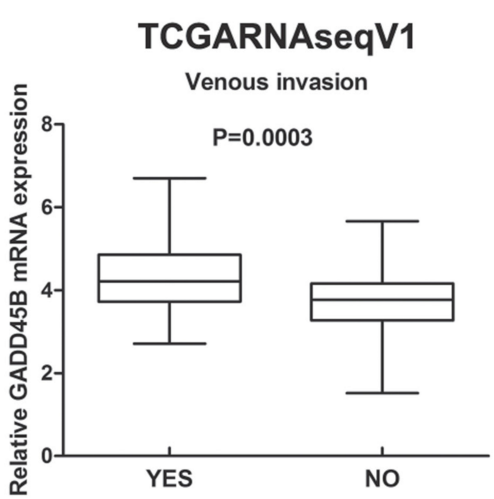

D

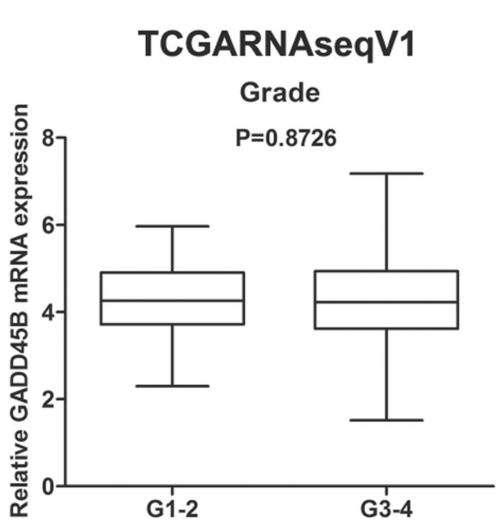

G

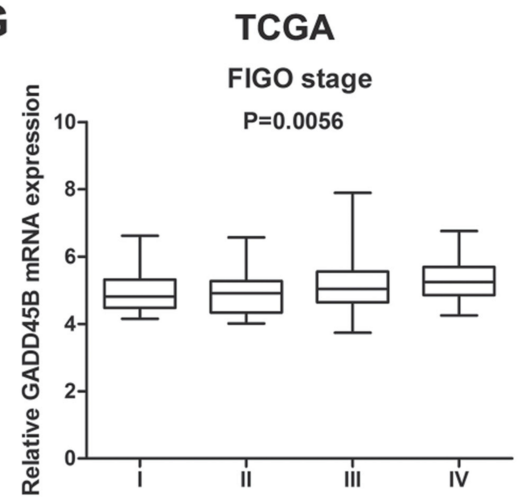

B

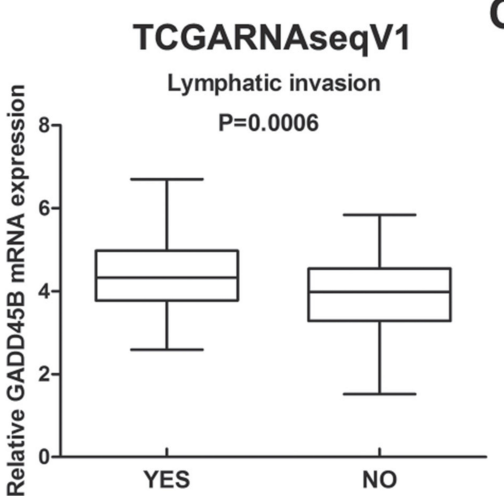

E TCGA

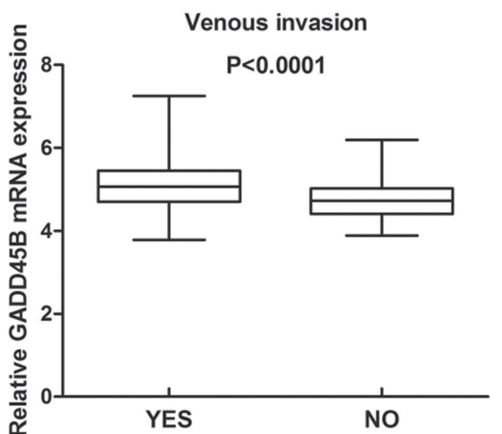

H

TCGA

Grade

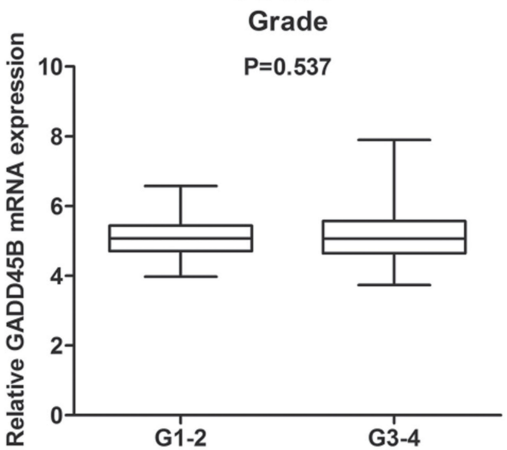

F

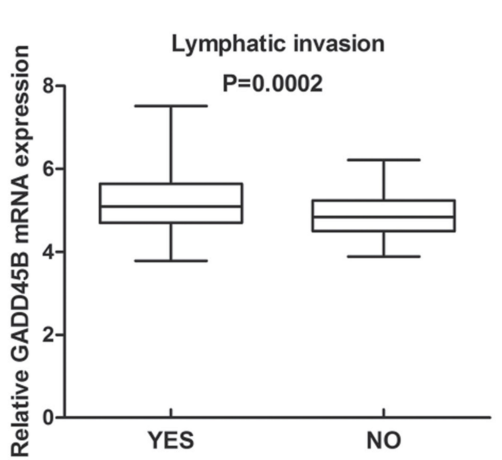

C

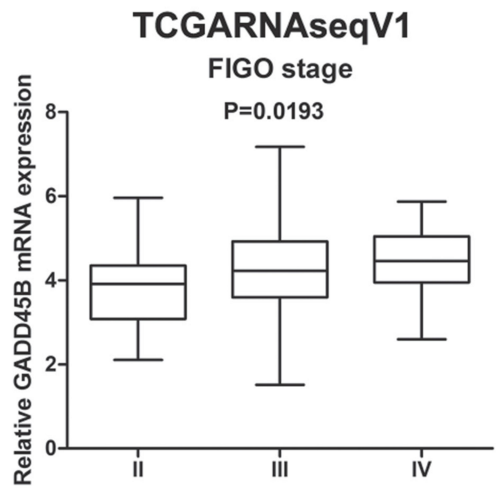

TCGA

I GSE49997 peritoneal carcinomatosis

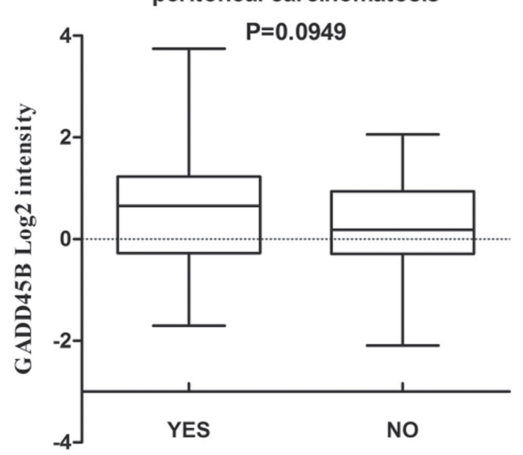

Figure 2 High GADD45B mRNA levels are related to metastasis of EOC. The relationship between GADD45B mRNA levels and clinicopathological features in TCGA and GSE49997 cohorts. (A, E) The relationship between GADD45B expression and venous invasion. (B, F) The relationship between GADD45B expression and lymphatic invasion. (C, G) The relationship between GADD45B expression and FIGO stage. (I) The relationship between GADD45B expression and peritoneal carcinomatosis. (D, H) The relationship between GADD45B expression and grade. The results are shown as the mean \pm s.d. Student's $t$-test was used to analyse the data.

pathway among the "hallmark signature" gene sets (P value $<0.05$ ) (Figure 5C). Finally, the correlation of GADD45B mRNA expression and EMT markers was calculated in the TCGA cohort. As shown in
Figure 5D, GADD45B was negatively related to CDH1 (E-cadherin) but positively related to SNAIL1, SNAIL2, TWIST1, ZEB1 and ZEB2, which meant that $G A D D 45 B$ correlates with EMT in EOC. 
A

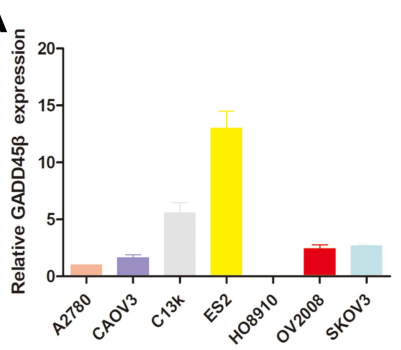

B

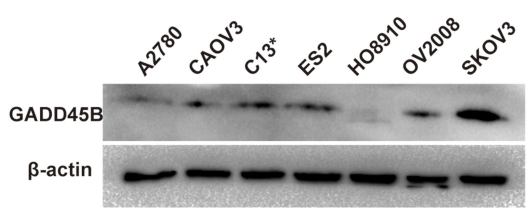

C

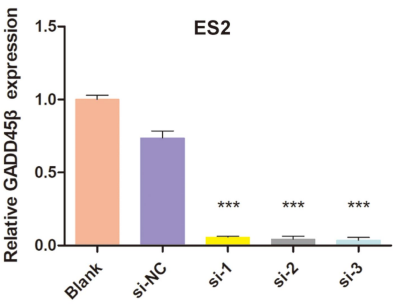

D

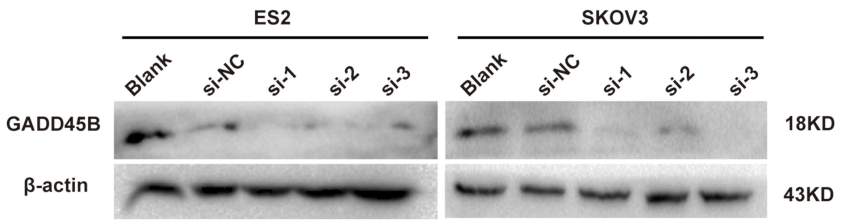

E

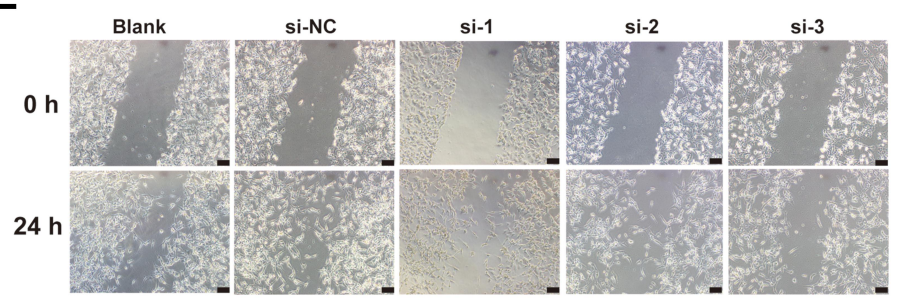

G

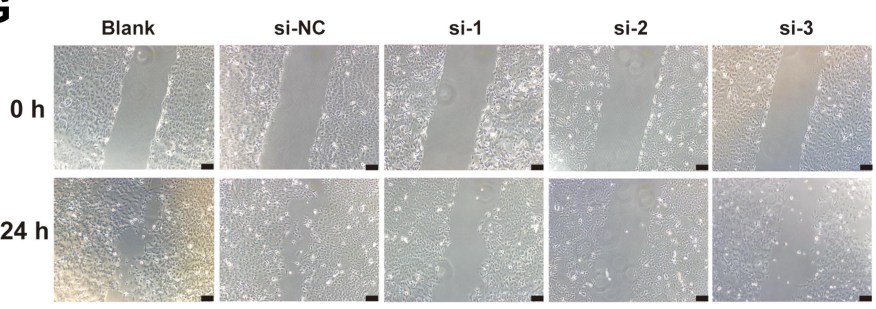

I

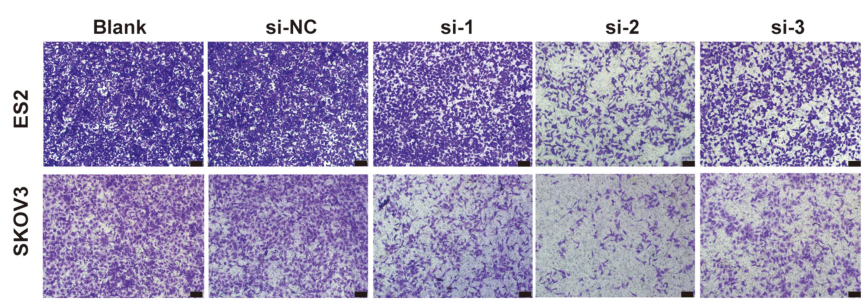

K

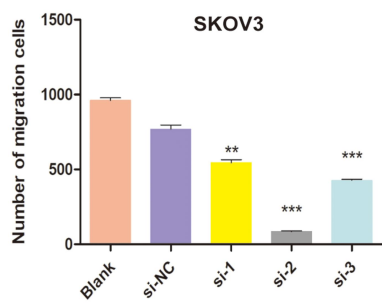

$\mathbf{F}$

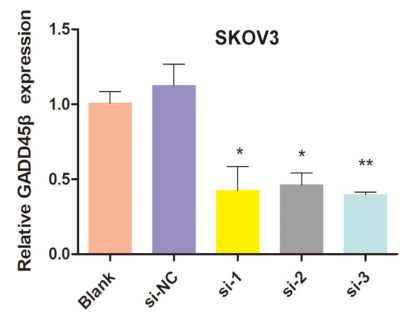

H

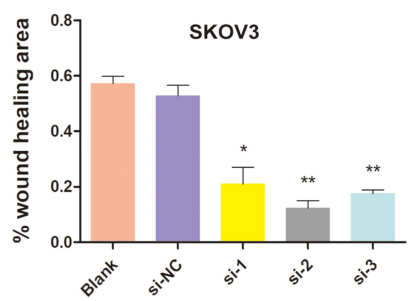

J

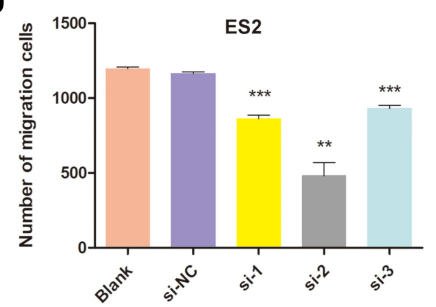

Figure 3 GADD45B knockdown inhibited the migration of EOC cells. (A, B) Western blot and qRT-PCR detected the expression of GADD45B in the EOC cell lines. (C, D) qRT-PCR and Western blot analysis of the silencing efficiency of GADD45B. (E, G) Wound-healing assays detected the migration ability difference of ES2 and SKOV3 cells between the NC group and the GADD45B siRNA-downregulated groups. (F, H) Histograms show the wound-healing areas of the scratch-wound assays. (I) Transwell assays detected the migration ability of ES2 and SKOV3 cells in the NC group and the GADD45B siRNA-downregulated groups. (J, K) Histograms show the number of migrating cells. Experiments were performed at least in triplicate, and the results are shown as the mean \pm s.d. Student's $t$-test was used to analyse the data $\left({ }^{*} \mathrm{P}<0.05\right.$; ${ }^{* *} \mathrm{p}<0.0 \mathrm{I} ;{ }^{* * *} \mathrm{P}$ $<0.00 \mathrm{I})$. Scale bar, $100 \mu \mathrm{m}$. 
A
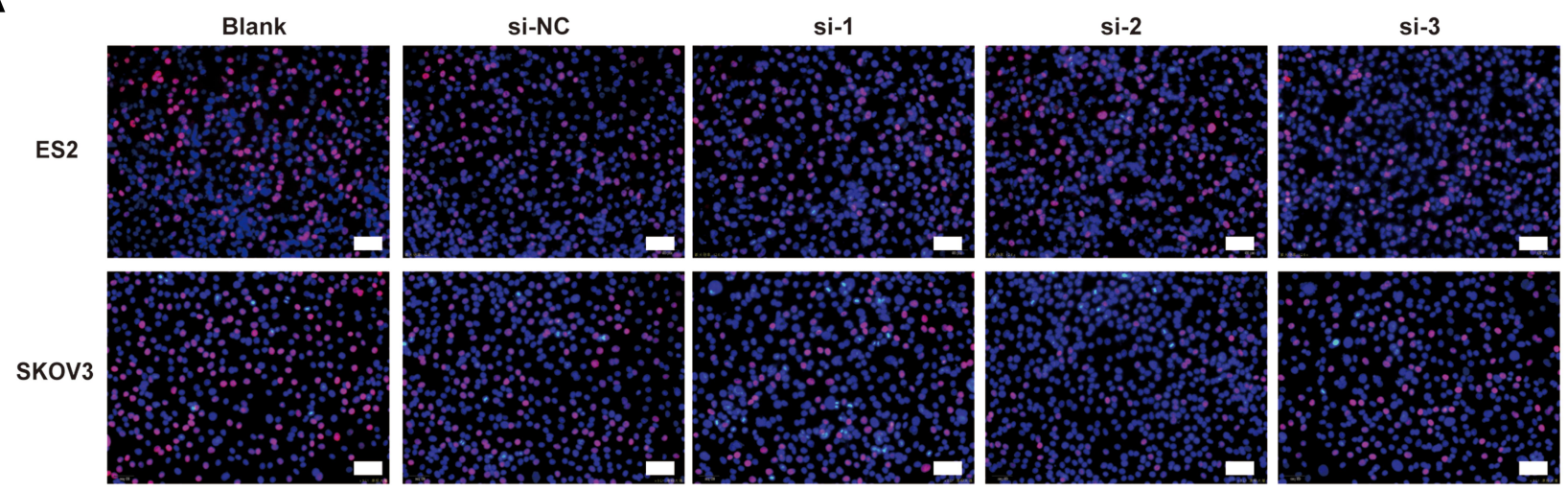

B

ES2

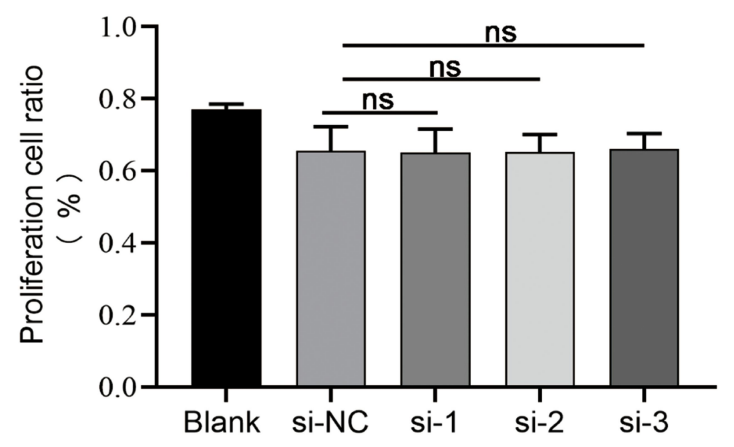

C

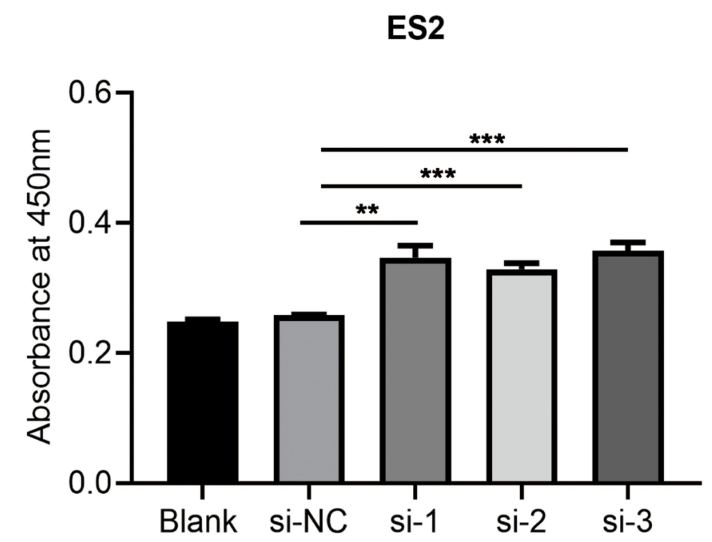

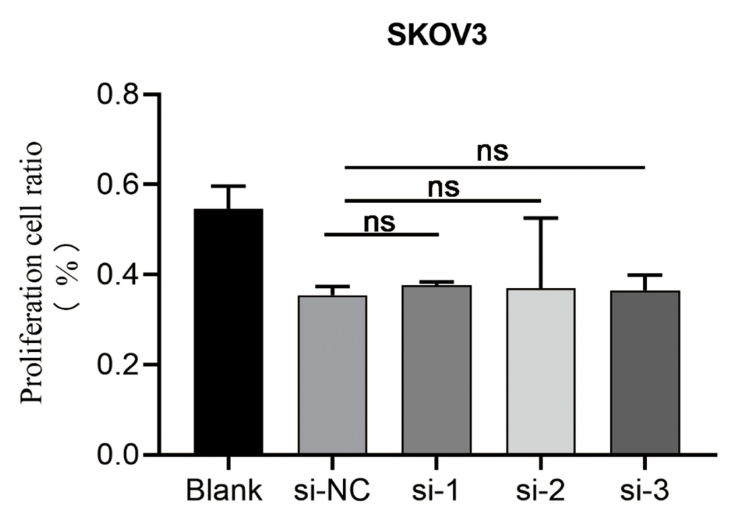

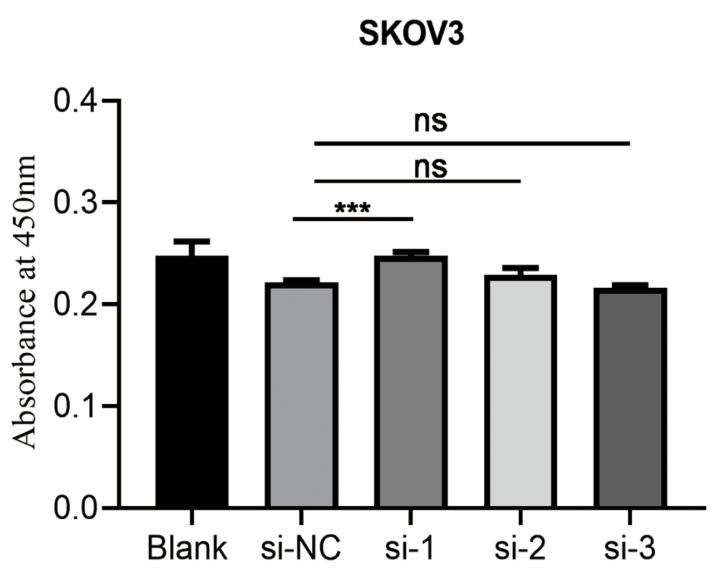

Figure 4 GADD45B knockdown does not inhibit proliferation and viability of EOC cells. (A) EdU assays detected the proliferation ability of ES2 and SKOV3 cells in the NC group and the GADD45B siRNA-downregulated groups. (B) Histograms show the proliferation cell ratio of the EdU assays. (C) CCK-8 assays detected the viability difference of ES2 and SKOV3 cells between the NC group and the GADD45B siRNA-downregulated groups. Experiments were performed at least in triplicate, and the results are shown as the mean \pm s.d. Student's $t$-test was used to analyse the data (ns, not significant; $* * p<0.01$; $* * * p<0.00 I$ ). Scale bar, I00 $\mu$ m.

\section{GADD45B Promotes the Migration of EOC Cells Through EMT}

To further validate the effect of $G A D D 45 B$ on EMT in ovarian cancer cells, we detected the expression of EMT markers after GADD45B knockdown. The slender ES2 and SKOV3 cells became short and round (Figure 6A) when GADD45B expression was decreased by siRNA. Compared with the NC group, the three siRNA treatments significantly 
A

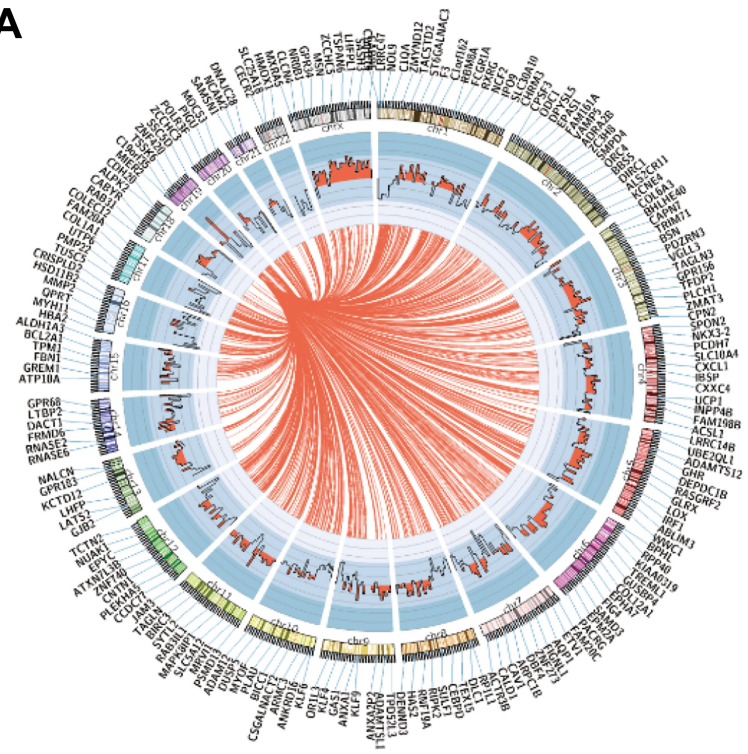

C

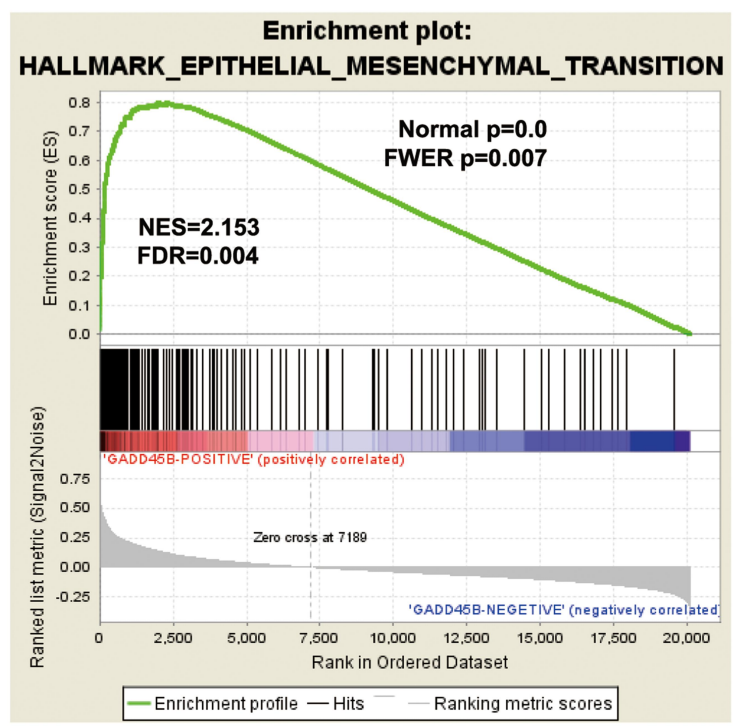

B

KEGG parhway enrichment of GADD45B coexpression genes

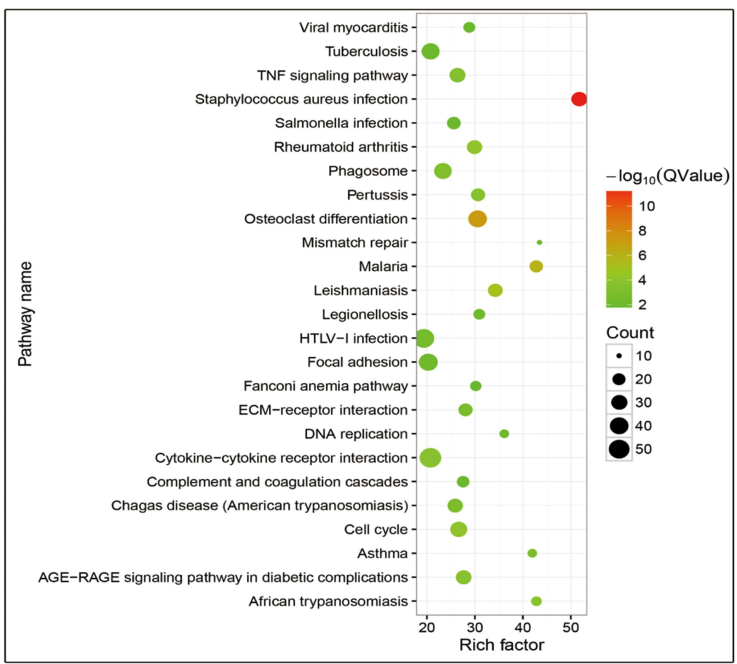

D
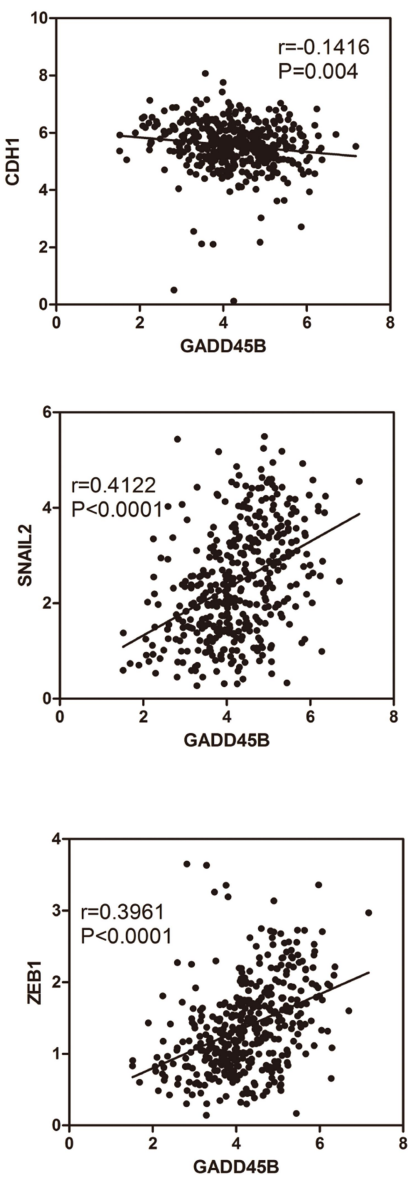
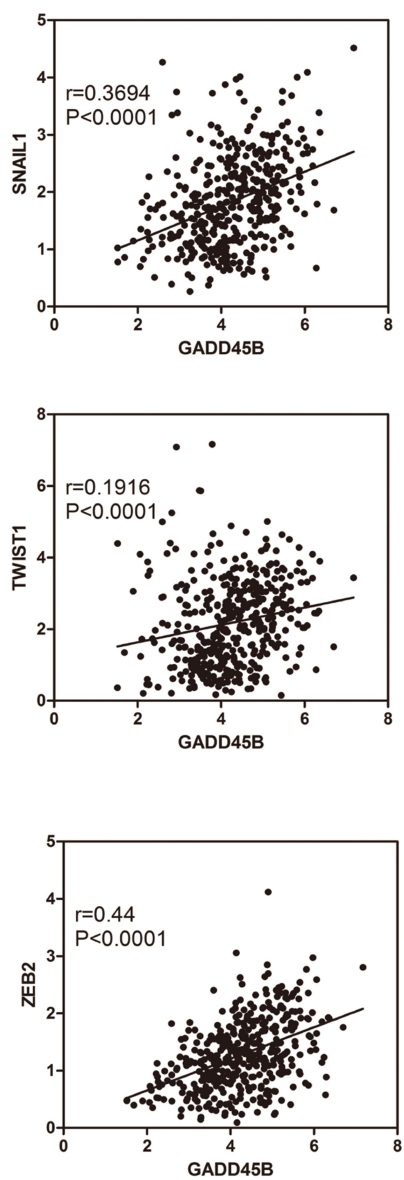

Figure 5 GADD45B correlates with the epithelial-mesenchymal transition of EOC. (A) The correlated genes of GADD45B are shown in the gene-label circular diagram. (B) KEGG signalling pathway analysis revealed significant pathway enrichment for GADD45B coexpressed genes. (C) The relationship between GADD45B expression levels and the epithelial-mesenchymal transition pathway was shown by GSEA analysis of the TCGA cohort. (D) The correlation of GADD45B mRNA expression and EMT markers was calculated in the TCGA cohort. 
A
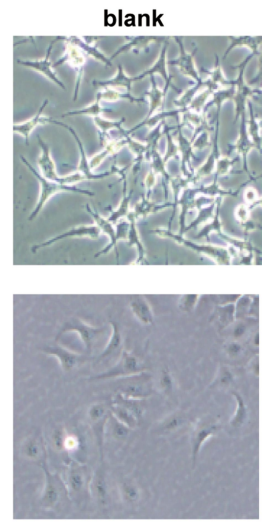

si-NC
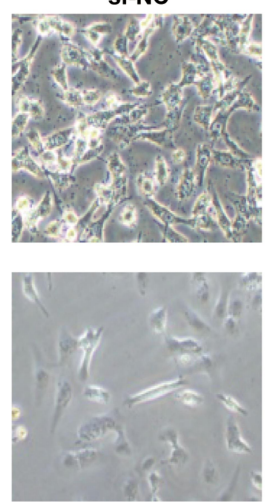

si-1
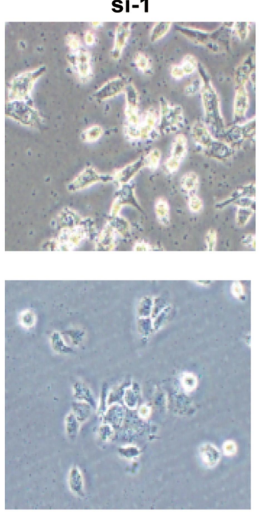
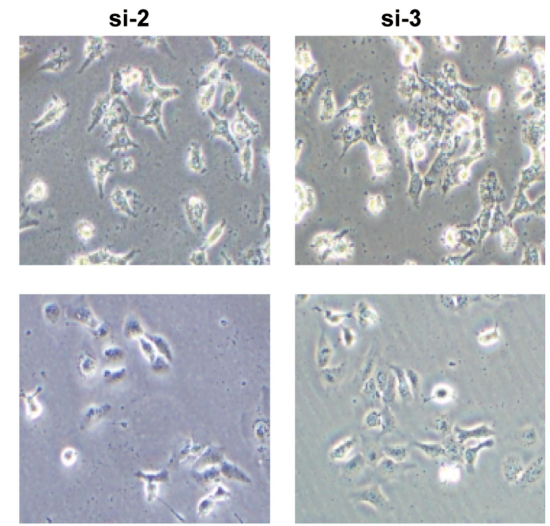

B

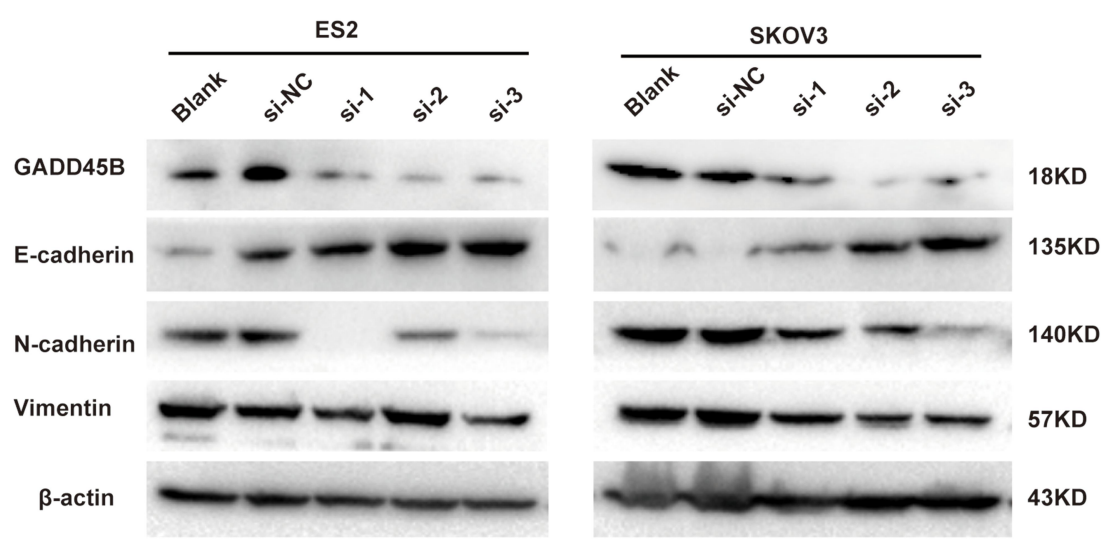

C

ES2

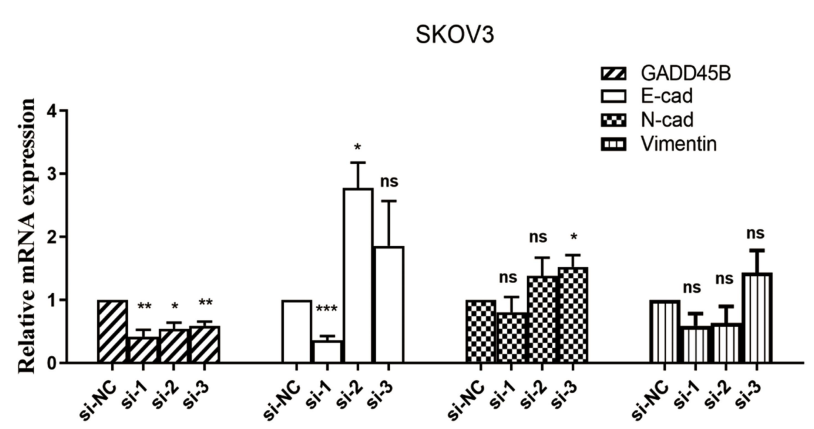

Figure 6 GADD45B promotes the migration of EOC cells through EMT. (A) The morphology of the ES2 and SKOV3 OC cell lines transfected with GADD45B or NC siRNA was observed under a microscope. (B) Western blot analysis of the expression of E-cadherin, N-cadherin and vimentin in ES2 and SKOV3 cells after transfection with GADD45B siRNA or NC. (C) qRT-PCR detected the expression of E-cadherin, N-cadherin and vimentin in ES2 and SKOV3 cells after transfection of GADD45B siRNA or NC. Experiments were performed at least in triplicate, and the results are shown as the mean \pm s.d. Student's $t$-test was used to analyse the data (ns, not significant; ${ }^{*} \mathrm{p}<0.05$; ${ }^{* *} \mathrm{p}$ $<0.01 ; * * * p<0.001)$.

downregulated the protein expression level of GADD45B, and the downregulation of GADD45B increased the protein level of E-cadherin but decreased that of $\mathrm{N}$-cadherin and Vimentin (Figure 6B). The mRNA level was not consistent with the protein level (Figure 6C). These results suggested that $G A D D 45 B$ promoted the migration of ovarian cancer cells through the EMT pathway.

\section{GADD45B is Overexpressed in EOC Omental Metastasis Tissues Over Matched Primary Tumour Tissues}

To further investigate the correlation of GADD45B in ovarian cancer metastasis, we detected the protein expression level of GADD45B in 11 matched pairs of ovarian cancer primary tumour tissues and omental metastasis tissue 
samples through immunohistochemical staining of serial sections. The expression of GADD45B was detected in all tumour tissues, and the expression level was higher in omental metastasis tissues than in primary tumour tissues in 11 pairs of matched tissue samples (Figure 7A and B). All patient clinical information is shown in Table 3. In summary, our results suggest that $G A D D 45 B$ can promote the invasion and migration of ovarian cancer cells through EMT and is associated with ovarian cancer metastasis.

\section{Discussion}

Herein, our work demonstrates for the first time that elevated $G A D D 45 B$ correlates significantly with metastasis and poor EOC patient survival through several large cohorts of TCGA and GEO databases. We validated that $G A D D 45 B$ facilitates the motility of epithelial ovarian cancer through the EMT pathway and is correlated with tumour metastasis. Thus, GADD 45B may be a new biomarker of metastasis and prognosis

A

Patient 10\#

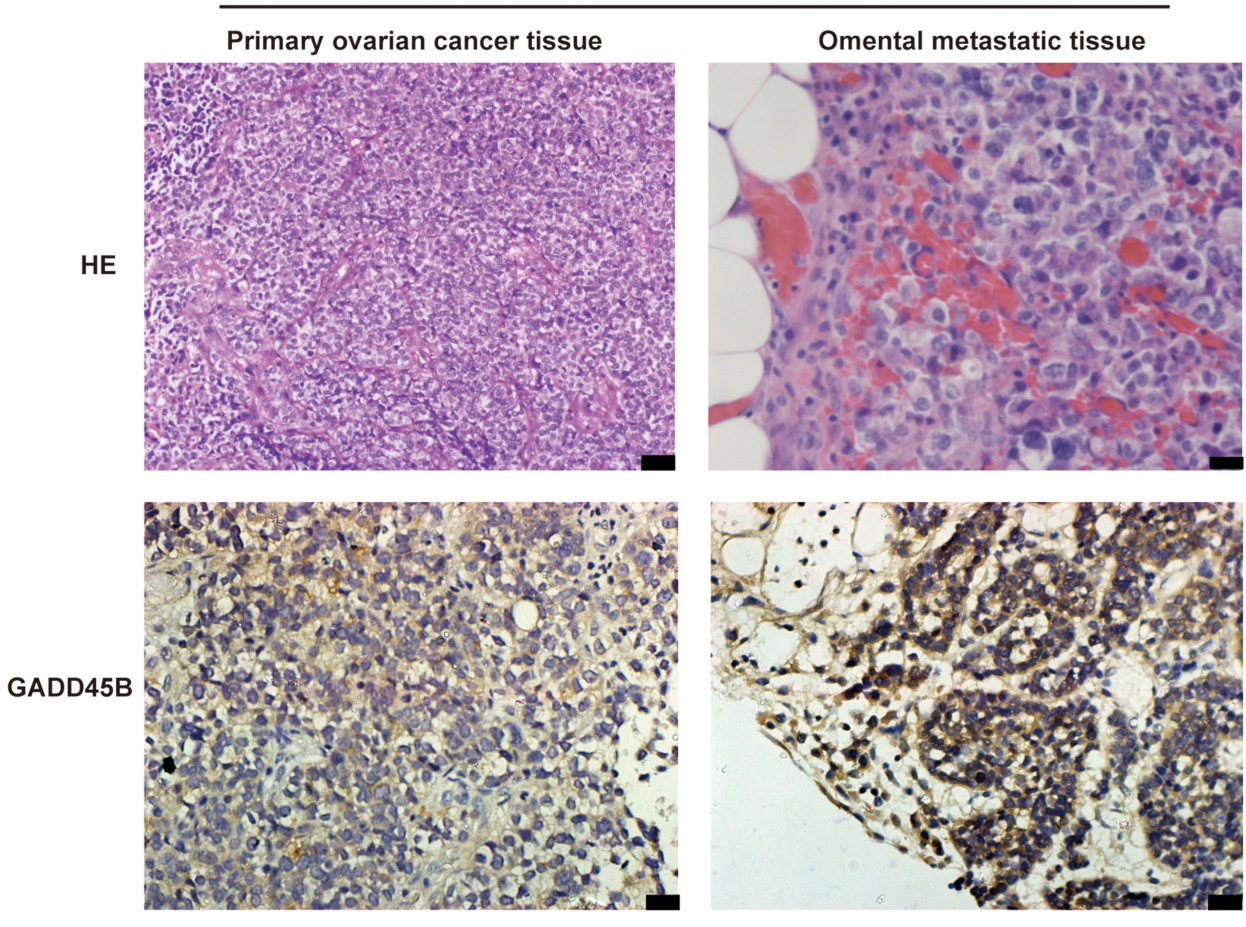

B

GADD45B

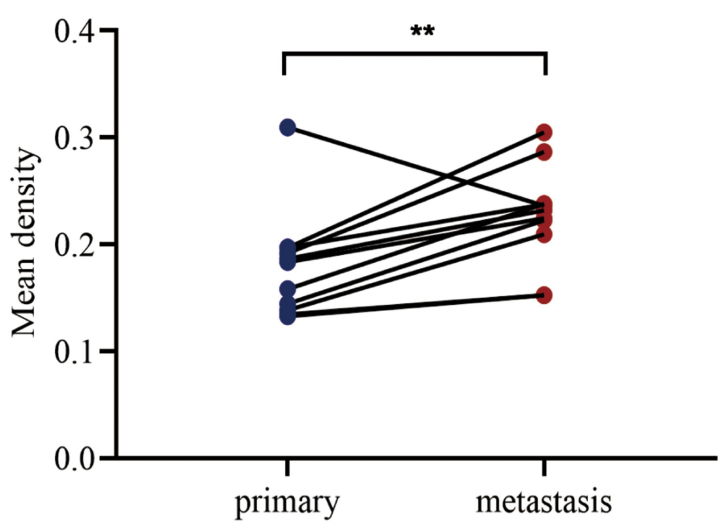

Figure 7 GADD45B is overexpressed in omental metastatic tissues of EOC over matched primary tumour tissues. (A) Representative images of the immunohistochemical analysis of GADD45B in one of the paired tumour tissues. Scale bar, $20 \mu \mathrm{m}$. (B) Statistical result of the mean density of GADD45B in II pairs of matched EOC tissue samples. Paired Student's $t$-test was used to analyse the data $\left({ }^{* *} \mathrm{p}<0.01\right)$. 
Table 3 Clinicopathological Features of the EOC Patients Studied by Immunohistochemistry

\begin{tabular}{|c|c|c|c|c|c|c|c|}
\hline \multirow{2}{*}{$\begin{array}{l}\text { Patient } \\
\text { No. }\end{array}$} & \multirow{2}{*}{$\begin{array}{l}\text { Pathological } \\
\text { Type }\end{array}$} & \multirow[t]{2}{*}{ FIGO Stage } & \multirow{2}{*}{$\begin{array}{l}\text { Age } \\
\text { (Years) }\end{array}$} & \multirow{2}{*}{$\begin{array}{l}\text { Prior } \\
\text { Treatment }\end{array}$} & \multicolumn{3}{|c|}{ Peritoneal Metastasis } \\
\hline & & & & & $\begin{array}{l}\text { Fallopian } \\
\text { Tube }\end{array}$ & Omentum & Appendix \\
\hline I & HGSOC & IV & 47 & No & Yes & Yes & No \\
\hline 2 & HGSOC & IIIC & 63 & No & Yes & Yes & No \\
\hline 3 & HGSOC & IIIC & 38 & No & Yes & Yes & No \\
\hline 4 & HGSOC & IIIC & 67 & No & Yes & Yes & No \\
\hline 5 & HGSOC & IIIB & 55 & No & Yes & Yes & No \\
\hline 6 & HGSOC & IIIC & 65 & No & Yes & Yes & No \\
\hline 7 & HGSOC & IIIC & 55 & No & Yes & Yes & Yes \\
\hline 8 & HGSOC & IIIC & 58 & No & Yes & Yes & No \\
\hline 9 & HGSOC & IIIB & 51 & No & Yes & Yes & No \\
\hline 10 & HGSOC & IIIC & 51 & No & Yes & Yes & Yes \\
\hline II & HGSOC & IIIC & 73 & No & Yes & Yes & No \\
\hline
\end{tabular}

Abbreviations: EOC, epithelial ovarian cancer; FIGO, International Federation of Gynecology and Obstetrics; HGSOC, high-grade serous ovarian carcinoma.

and is expected to be a candidate therapeutic target for EOC.

$G A D D 45 B$ is important in stress signalling in response to physiological and environmental stress. ${ }^{20}$ Evidence has been obtained indicating that $G A D D 45 B$ can function to either promote or suppress tumour development, which is dependent on the molecular nature of the activated oncogene and the cell type, via engagement of different signalling pathways. $^{7}$ For instance, GADD $45 B$ presents as oncogenic and indicates poor survival in colorectal cancer, ${ }^{10}$ papillary thyroid carcinoma ${ }^{11}$ and human embryonic carcinoma. ${ }^{12}$ In contrast, GADD45B functions as a tumour suppressor of BCR-ABL-driven leukaemia, ${ }^{21}$ pituitary gonadotrope tumours ${ }^{22}$ and lymphoma. ${ }^{23}$ Here, we found that overexpression of $G A D D 45 B$ indicated poor EOC survival and was significantly associated with high FIGO stage, venous invasion and lymphatic invasion, suggesting that $G A D D 45 B$ is a promotive factor in EOC progression. Although opposite functions of GADD45B in different cancers have been observed, high expression of $G A D D 45 B$ was found to denote aggressive malignant disease and poor patient survival across most human cancers, ${ }^{16}$ and these results seemed contradictory. Recently, Daniela et al found that GADD45B inhibition in myeloid cells restored the activation of proinflammatory tumourassociated macrophages and intratumoural immune infiltration, thereby diminishing oncogenesis in various models of solid cancers refractory to immunotherapies, which may explain the paradoxical results. ${ }^{16}$ In addition, we verified the effect of $G A D D 45 B$ on EOC prognosis not only in TCGA but also in GEO cohorts, different from the reported pure bioinformatics analysis about the influence of GADD $45 B$ on EOC prognosis. ${ }^{14,15}$ Extending these studies, we validated the promotive impact on EOC migration in vitro and discovered that GADD45B knockdown inhibited the migration of ovarian cancer cells. Moreover, our immunohistochemical staining of the matched pairs of ovarian cancer primary tumour tissues and omental metastasis tissues validated that GADD45B expression was higher in omental metastasis tissues.

In this study, we revealed a new role for $G A D D 45 B$ in contributing to epithelial ovarian cancer progression by EMT. This is consistent with the reported function of GADD $45 B$ in cholangiocarcinoma. ${ }^{13}$ KEGG analysis of GADD $45 B$ coexpressed genes revealed that $G A D D 45 B$ was closely associated with the cell cycle, ECM-receptor interaction, focal adhesions, and TNF signalling pathway (Figure 5B), which are important functions involved in the EMT process. ${ }^{24}$ Further GSEA of the TCGA cohort showed that GADD45B expression was also strongly related to the EMT pathway. The overlap of the biological functions of $G A D D 45 B$ and EMT supports this conclusion. That is, GADD45B is an important modulator in 
cell cycle arrest, DNA repair, cell survival, and apoptosis, ${ }^{25-27}$ while cancer cells that acquire a mesenchymal phenotype after EMT progression display resistance to apoptosis, stem cell characteristics and tissue invasiveness. ${ }^{28,29}$ Furthermore, we found that $G A D D 45 B$ was negatively related to $C D H 1$ (E-cadherin) mRNA expression but positively related to SNAIL1, SNAIL2, TWIST1, ZEB1 and ZEB2 in the TCGA cohort (Figure 5D). These results coincide exactly with the perception that EMT is a complex, multifactorial program that is orchestrated and coordinated by a series of master EMTinducing transcription factors, notably SNAIL, SLUG, TWIST and ZEB1. ${ }^{30,31}$

GADD45B can function to either promote or suppress tumour development through different signalling pathways. ${ }^{7}$ $G A D D 45 B$ binds to the N-terminal domain of MTK1 (MEKK4) and mediates activation of the p38 and JNKMAPK (c-Jun kinase-mitogen-activated protein kinase) cascades in response to environmental stresses. ${ }^{8} G A D D 45 B$ elevated by NF- $\kappa \mathrm{B}$ binds to MKK7 and promotes multiple myeloma cell survival by blocking proapoptotic MKK7/JNK signalling. ${ }^{32-34}$ On the other hand, the p38 and MAPK pathways are well known for their close relationship with EMT. $^{35-37}$ Accordingly, MAPK pathway regulation may be the mechanism by which $G A D D 45 B$ facilitates EMT. $G A D D 45 B$ is an effector of transforming growth factor- $\beta$ (TGF- $\beta$ )-induced apoptosis, and $T G F-\beta$ induces $G A D D 45 B$ expression in a SMAD-dependent manner, activates $\mathrm{p} 38$ and triggers apoptotic cell death. ${ }^{38}$ Considering the important promotive function of the TGF- $\beta$ signalling pathway on EMT and metastasis in late-stage cancers, ${ }^{39,40}$ especially in EOC, ${ }^{41}$ we can speculate that $G A D D 45 B$ may be stimulated by $T G F-\beta$ and attributed to EMT in ovarian cancer through the MAPK pathway, which requires further investigation. Moreover, in this study, we found that the changes in the mRNA expression of E-cadherin, $\mathrm{N}$-cadherin and Vimentin were not as significant as their protein expression changes, which was consistent with the abovementioned cascades and indicates another unknown type of regulation or epigenetic regulation of $G A D D 45 B$ on downstream factors, ultimately promoting EMT in epithelial ovarian cancer.

\section{Ethical Statement}

The study was conducted according to the principles expressed in the Declaration of Helsinki and was approved by the Research Ethics Committee of Union Hospital, Tongji Medical College, Huazhong University of Science and Technology (Wuhan, China).

\section{Acknowledgments}

We would like to thank Mr. Guodong Li (Huazhong University of Science and Technology, Wuhan, China) for help with the bioinformatics analysis.

\section{Funding}

This work was supported by the National Natural Science Foundation of China (No. 81903012) and the Natural Science Foundation of Hubei Province (No. 2017CFB753).

\section{Disclosure}

The authors declare no competing financial or nonfinancial interests.

\section{References}

1. Jayson GC, Kohn EC, Kitchener HC, Ledermann JA. Ovarian cancer. The Lancet. 2014;384(9951):1376-1388.

2. Allemani C, Matsuda T, Di Carlo V, et al. Global surveillance of trends in cancer survival 2000-14 (CONCORD-3): analysis of individual records for 37513025 patients diagnosed with one of 18 cancers from 322 population-based registries in 71 countries. The Lancet. 2018.

3. Bray F, Ferlay J, Soerjomataram I, Siegel RL, Torre LA, Jemal A. Global cancer statistics 2018: GLOBOCAN estimates of incidence and mortality worldwide for 36 cancers in 185 countries. CA Cancer J Clin. 2018;68(6):394-424.

4. Torre LA, Trabert B, DeSantis CE, et al. Ovarian cancer statistics, 2018. CA Cancer J Clin. 2018;68(4):284-296.

5. Bowtell DD, Böhm S, Ahmed AA, et al. Rethinking ovarian cancer II: reducing mortality from high-grade serous ovarian cancer. Nat Rev Cancer. 2015;15(11):668-679.

6. Weidle UH, Birzele F, Kollmorgen G, Rueger R. Mechanisms and targets involved in dissemination of ovarian cancer. Cancer Genomics Proteomics. 2016;13(6):407-423.

7. Liebermann DA, Tront JS, Sha X, Mukherjee K, Mohamed-Hadley A, Hoffman B. Gadd45 stress sensors in malignancy and leukemia. Crit Rev Oncog. 2011;16(1-2):129-140.

8. Takekawa M, Saito H. A family of stress-inducible GADD45-like proteins mediate activation of the stress-responsive MTK1/MEKK4 MAPKKK. Cell. 1998;95(4):521-530.

9. Kigar SL, Chang L, Auger AP. Gadd45b is an epigenetic regulator of juvenile social behavior and alters local pro-inflammatory cytokine production in the rodent amygdala. Brain Behav Immun. 2015;46:60-69.

10. Zhao Z, Gao Y. GADD45B as a prognostic and predictive biomarker in Stage II colorectal cancer. 2018;9:7.

11. Barros-Filho MC, de Mello JBH, Marchi FA, et al. GADD45B transcript is a prognostic marker in papillary thyroid carcinoma patients treated with total thyroidectomy and radioiodine therapy. Front Endocrinol (Lausanne). 2020;11:269.

12. Inowa T, Hishikawa K, Matsuzaki Y, et al. GADD45 $\beta$ determines chemoresistance and invasive growth of side population cells of human embryonic carcinoma. Stem Cells Int. 2010;2010:782967.

13. Myint KZ, Kongpracha P, Rattanasinganchan P, et al. Gadd $45 \beta$ silencing impaired viability and metastatic phenotypes in cholangiocarcinoma cells by modulating the EMT pathway. Oncol Lett. 2018;15(3):3031-3041. 
14. Li L, Cai S, Liu S, Feng H, Zhang J. Bioinformatics analysis to screen the key prognostic genes in ovarian cancer. $J$ Ovarian Res. 2017;10(1):27.

15. Lu X, Lu J, Liao B, Li X, Qian X. Driver pattern identification over the gene co-expression of drug response in ovarian cancer by integrating high throughput genomics data. 2017;7(1):16188.

16. Verzella D, Bennett J, Fischietti M, et al. GADD45 $\beta$ loss ablates innate immunosuppression in cancer. Cancer Res. 2018;78 (5):1275-1292.

17. Pils D, Hager G, Tong D, et al. Validating the impact of a molecular subtype in ovarian cancer on outcomes: a study of the OVCAD Consortium. Cancer Sci. 2012;103(7):1334-1341.

18. Gyorffy B, Lánczky A, Szállási Z. Implementing an online tool for genome-wide validation of survival-associated biomarkers in ovarian-cancer using microarray data from 1287 patients. Endocr Relat Cancer. 2012;19(2):197-208.

19. Guo J, Cai J, Zhang Y, Zhu Y, Yang P, Wang Z. Establishment of two ovarian cancer orthotopic xenograft mouse models for in vivo imaging: a comparative study. Int J Oncol. 2017;51(4):1199-1208.

20. Takekawa M, Saito HA. Family of Stress-Inducible GADD45-like proteins mediate activation of the stress-responsive MTK1/MEKK4 MAPKKK. Cell. 1998;95(4):521-530.

21. Sha X, Hoffman B, Liebermann DA. Loss of Gadd45b accelerates BCR-ABL-driven CML. Oncotarget. 9(70):33360-33367.

22. Michaelis KA, Knox AJ, Xu M, et al. Identification of growth arrest and DNA-damage-inducible Gene $\beta$ (GADD45 $\beta$ ) as a novel tumor suppressor in pituitary gonadotrope tumors. Endocrinology. 2011;152 (10):3603-3613.

23. Engelmann A, Speidel D, Bornkamm GW, Deppert W, Stocking C. Gadd $45 \beta$ is a pro-survival factor associated with stress-resistant tumors. Oncogene. 2008;27(10):1429-1438.

24. Dongre A, Weinberg RA. New insights into the mechanisms of epithelial-mesenchymal transition and implications for cancer. Nat Rev Mol Cell Biol 2019/02/01. 2019;20(2):69-84.

25. Tang XH, Li H, Zheng XS, Lu MS. CRM197 reverses paclitaxel resistance by inhibiting the NAC-1/Gadd45 pathway in paclitaxel-resistant ovarian cancer cells. 2019;8(14):6426-6436.

26. Yang TC, Wu PC, Chung IF, Jiang JH, Fann MJ, Kao LS. Cell death caused by the synergistic effects of zinc and dopamine is mediated by a stress sensor gene Gadd45b - implication in the pathogenesis of Parkinson's disease. J Neurochem. 2016;139(1):120-133.

27. Lucas A, Mialet-Perez J, Daviaud D, Parini A, Marber MS, Sicard P. Gadd $45 \gamma$ regulates cardiomyocyte death and post-myocardial infarction left ventricular remodelling. Cardiovasc Res. 2015;108 (2):254-267.
28. Mani SA, Guo W, Liao MJ, et al. The epithelial-mesenchymal transition generates cells with properties of stem cells. Cell. 2008;133 (4):704-715

29. Georgakopoulos-Soares I, Chartoumpekis DV, Kyriazopoulou V, Zaravinos A, Factors EMT. Metabolic pathways in cancer. Front Oncol. 2020;10:499.

30. Craene BD, Berx G. Regulatory networks defining EMT during cancer initiation and progression. Nat Rev Cancer. 2013;13(2):97-110.

31. Lamouille S, $\mathrm{Xu}$ J, Derynck R. Molecular mechanisms of epithelial-mesenchymal transition. Nat Rev Mol Cell Biol. 2014;15 (3):178-196.

32. De Smaele E, Zazzeroni F, Papa S, et al. Induction of gadd $45 \beta$ by $\mathrm{NF}-\kappa \mathrm{B}$ downregulates pro-apoptotic JNK signalling. Nature. 2001;414(6861):308-313.

33. Tornatore L, Sandomenico A, Raimondo D, et al. Cancer-selective targeting of the NF- $\mathrm{BB}$ survival pathway with GADD45 $\beta / \mathrm{MKK} 7$ inhibitors. Cancer Cell. 2014;26(4):495-508.

34. Papa S, Zazzeroni F, Bubici C, et al. Gadd $45 \beta$ mediates the NF- $\kappa B$ suppression of JNK signalling by targeting MKK7/JNKK2. Nat Cell Biol. 2004;6(2):146-153.

35. Kuo CL, Chou HY, Chiu YC, et al. Mitochondrial oxidative stress by Lon-PYCR1 maintains an immunosuppressive tumor microenvironment that promotes cancer progression and metastasis. Cancer Lett. 2020;474:138-150.

36. Xu J, Shi J, Tang W. ROR2 promotes the epithelial-mesenchymal transition by regulating $\mathrm{MAPK} / \mathrm{p} 38$ signaling pathway in breast cancer. 2020;12.

37. Olea-Flores M, Zuñiga-Eulogio MD. Extracellular-signal regulated kinase: a central molecule driving epithelial-mesenchymal transition in cancer. 2019;20:12.

38. Yoo J, Ghiassi M, Jirmanova L, et al. Transforming growth factor-beta-induced apoptosis is mediated by Smad-dependent expression of GADD45b through p38 activation. J Biol Chem. 2003;278(44):43001-43007.

39. Massagué J. TGFbeta in Cancer. Cell. 2008;134(2):215-230.

40. Teixeira AF, Ten Dijke P, Zhu HJ. On-target Anti-TGF- $\beta$ therapies are not succeeding in clinical cancer treatments: what are remaining challenges? Front cell Develop Biol. 2020;8:605.

41. Roane BM, Arend RC, Birrer MJ. Review: targeting the transforming growth factor-beta pathway in ovarian cancer. Cancers. 2019;11:5.
OncoTargets and Therapy

\section{Publish your work in this journal}

OncoTargets and Therapy is an international, peer-reviewed, open access journal focusing on the pathological basis of all cancers, potential targets for therapy and treatment protocols employed to improve the management of cancer patients. The journal also focuses on the impact of management programs and new therapeutic

Submit your manuscript here: https://www.dovepress.com/oncotargets-and-therapy-journa agents and protocols on patient perspectives such as quality of life, adherence and satisfaction. The manuscript management system is completely online and includes a very quick and fair peer-review system, which is all easy to use. Visit http://www.dovepress.com/ testimonials.php to read real quotes from published authors. 